\begin{tabular}{|c|c|}
\hline Title & Low Reflectance Surface Observed on InP Porous Structures after Photoelectrochemical Etching \\
\hline Author(s) & Sato, Taketomo; Y oshizawa, Naoki; Okazaki, Hiroyuki; Hashizume, Tamotsu \\
\hline Citation & $\begin{array}{l}\text { ECS Transactions, 25(42), } 83-88 \\
\text { https://doi.org/10.1149/1.3416205 }\end{array}$ \\
\hline Issue Date & 2010 \\
\hline Doc URL & http:/hdl.handle.net/2115/48288 \\
\hline Rights & $\begin{array}{l}\text { () The Electrochemical Society, Inc. 2010. All rights reserved. Except as provided under U.S. copyright law, this work } \\
\text { may not be reproduced, resold, distributed, or modified without the express permission of The Electrochemical Society } \\
\text { (ECS). The archival version of this work was published in ECS T rans., 25(42), pp. 83-88. }\end{array}$ \\
\hline Type & article \\
\hline File Information & ECSt25-42_83-88.pdf \\
\hline
\end{tabular}

Instructions for use 


\title{
Low Reflectance Surface Observed on InP Porous Structures after Photoelectrochemical Etching
}

\author{
T. Sato, N. Yoshizawa, H. Okazaki, and T. Hashizume \\ Research Center for Integrate Quantum Electronics, Hokkaido University, \\ North 13, West 8, Sapporo 060-8628, Japan
}

\begin{abstract}
Extremely low reflectance was obtained from InP porous nanostructures in UV, visible, and near-infrared light ranges. The reflectance strongly depended on the surface morphology of the porous structures prepared by the electrochemical process, and the lowest reflectance of $0.1 \%$ in the visible light range was obtained from a sample after the irregular top layer was completely removed. Large anodic photocurrents were obtained on the InP porous structures that had low reflectance surfaces with deeper pores.
\end{abstract}

\section{Introduction}

InP is one of the most attractive materials for high-speed electronics and optoelectronic devices. Photosensitive devices such as photodetectors (PDs) in long-wavelength optical fiber communication systems $(1,2)$ and high-efficiency solar cells with multi-junction structures (3) are excellent examples of technologies that utilize the superb optical properties of InP-based systems. With the aforementioned devices, however, surface reflection is a serious problem that degrades the device performance because is obviously reduces the efficiency of a photon energy conversion (4). Surface texturing of V-grooves (5)-(7), formation of insulator films (8), wide bandgap semiconductor films (9), and transparent conducting oxide (TCO) films (10) have been investigated as anti-reflective (AR) layers on top of InP.

In this paper, we report that extremely low reflectance below $0.4 \%$ was observed from the InP porous nanostructures in UV, visible, and near-infrared ranges. The surface reflectance spectroscopy was carried out on the porous samples prepared using the electrochemical process, leading to finding that the reflectance strongly depended on the surface morphology. Photoelectrochemical measurements on the porous structures revealed that the photocurrents increased in the samples that had low reflectance surfaces with deeper pores.

\section{Experimental}

The porous samples were electrochemically formed on a layer of (001) n-type InP ( $\mathrm{n}=2$ $3 \times 10^{18} \mathrm{~cm}^{-3}$ ), on the back of which a GeAu/Ni-ohmic contact was formed for a current supply. The InP substrate was first anodized at $5 \mathrm{~V}$ in an HCl-based electrolyte to form the porous structures, where the structure depth, $d$, could be controlled by the anodization time, $t_{\mathrm{a}}(11)$. A disordered irregular layer formed and remained on top of the ordered porous layer after the first anodization. To remove this irregular layer, the porous surface was then photo-electrochemically etched under illumination at an anodic bias of $1 \mathrm{~V}$ in 
the same electrolyte (12). Figure 1(a) shows typical scanning electron microscope (SEM) images of the InP porous sample after photo-electrochemical (PEC) etching for $200 \mathrm{~s}$ $\left(t_{\mathrm{PEC}}=200 \mathrm{~s}\right)$. The average pore diameter in the sample before the PEC etching was about $30 \mathrm{~nm}$. On the other hand, regular sized pores about $130 \mathrm{~nm}$ in diameter appeared after PEC etching with $t_{\mathrm{PEC}}=200 \mathrm{~s}$, which was useful in completely removing the disordered layer from the surface. From the SEM observation on the cross-section, it was found that 130-nm-diameter nanopores were laterally separated by 50 -nm-thick InP nanowalls.

In this study, the reflectance spectra of samples were first characterized using an ultra-violet (UV)-visible spectrometer (UV-1700, Shimadzu). The photon energy range of the light source was set from 1.12 to $6.0 \mathrm{eV}$ corresponding to the wavelength range from 205 to $1100 \mathrm{~nm}$. After that, the photoelectrochemical measurements were conducted on the various porous InP electrodes under white light using a tungsten lamp. The experimental setup and the schematics for the porous electrode are outlined in Fig. 1(b). A series of electrochemical measurements was conducted using a standard cell with three electrodes: an InP porous electrode used as a working electrode (WE), a Pt counter electrode (CE), and a saturated calomel electrode (SCE) used as a reference. All electrodes were dipped into $1 \mathrm{mM} \mathrm{K}_{3} \mathrm{Fe}(\mathrm{CN})_{6}$ electrolyte. The potential of WE, $V_{\mathrm{s}}$, with respect to SCE was precisely controlled with a potentiostat with a voltage source. In this electrolyte system, n-type InP surface is relatively stable and a large potential barrier with the $\left[\mathrm{Fe}(\mathrm{CN})_{6}\right]^{3-} /^{4-}$ redox potential is expectable (13).

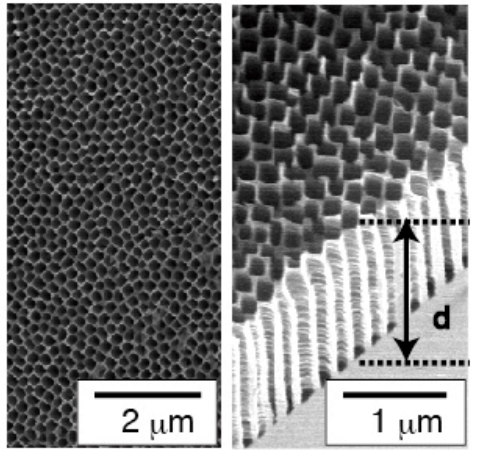

(a)
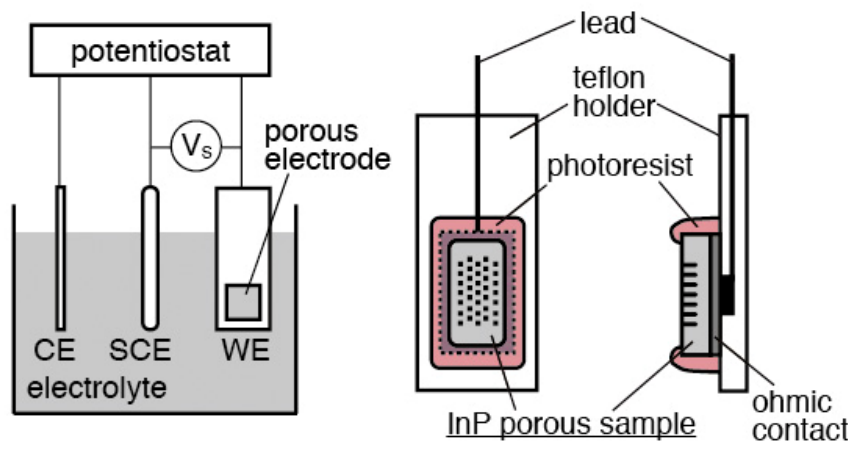

(b)

Figure 1. (a) SEM images of the porous sample after the complete removal of irregular top layers by PEC etching. (b) Schematic illustration of the electrochemical process and InP porous electrode used for the electrochemical measurements.

\section{Results and discussion}

We found that the optical view of the porous surface was reflected in the differences in the microscopic surface morphology. As shown in Fig. 2(a), the PEC etched porous sample has diffuse optical reflection resulting in a black surface. In order to clarify the optical reflectance on the porous structures, we investigated the effects of the irregular top layer on the surface reflectance by comparing three kinds of samples, as shown in Fig. 2(b). The pore depth, $d$, of the sample just after anodization without PEC etching was $13.8 \mu \mathrm{m}$, including the 4.0- $\mu \mathrm{m}$-thick irregular top layer. After the sample was PEC

etched for $200 \mathrm{~s}$, the $d$ decreased to $9.6 \mu \mathrm{m}$ due to the irregular layer being removed. First, 
the reflectance of the planar sample was higher than $30 \%$ over the measurement range. Typical peaks were observed around $1.4 \mathrm{eV}, 3.0 \mathrm{eV}$, and 4.5-5.6 eV, which were attributed to the interband transitions (14). The highest peak observed below $1.4 \mathrm{eV}$ was the reflection on the back ohmic contact, which acted as a mirror for light transmitted through the InP bandgap. The reflectance obtained from the porous sample without PEC etching was 5-10\% lower than that obtained from the reference sample. As plotted in Fig. 2(b), the spectral features of the InP bulk remained in the porous sample, showing that crystal quality was maintained beyond a certain level after the pore formed. On the other hand, the reflectance of the porous sample with the PEC etching drastically decreased compared with the other two samples. These results are very consistent with the optical view shown in Fig. 2(a). For the sample after the PEC etching, the reflectance was lower than $0.4 \%$ over the measurement range.

A possible explanation for the low reflectance observed here is that the straight nanopores with enlarged openings appeared on the surface after the PEC etching. In the present case with our porous samples, air holes are closely aligned between the InP nanowalls with a filling factor, $f$, of about 0.3 . This kind of air-dielectric composite, which when assembled in an ordered array, has a small $n$ close to unity leading to the low reflectance on the air interface. On the other hand, it should be considered that the irregular top layer has a large $n$ value due to the winding pores with poor porosity, resulting in the surface reflectance reducing by only a few percent.

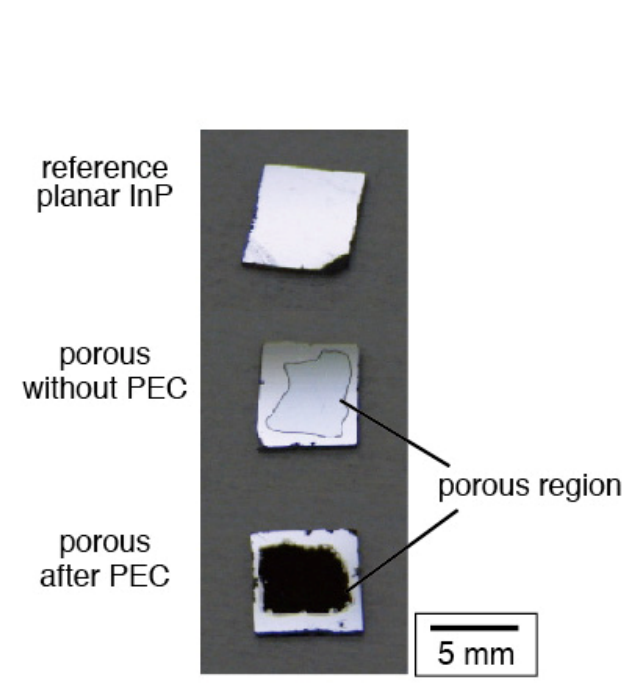

(a)

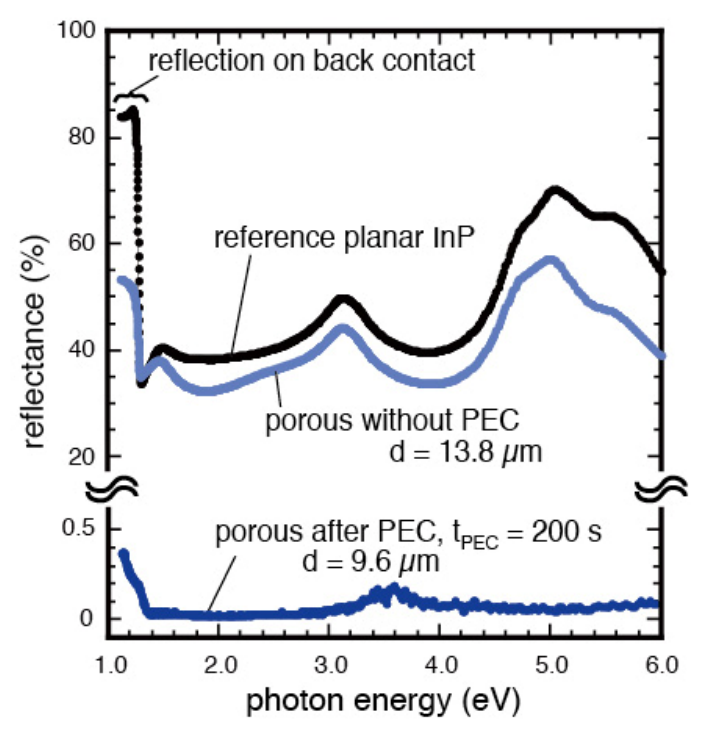

(b)

Figure 2. (a) Photograph of planar InP reference, porous sample without PEC etching, and porous sample after PEC etching. (b) Surface reflectance spectra measured as a function of photon energy of incident light for three samples.

The photoelectrochemical measurements were conducted on the InP porous structures after the irregular top layer had been removed. Figure 3(a) shows the cyclic voltammograms obtained at the porous InP electrodes with $d=9.6 \mu \mathrm{m}$ in the $\mathrm{K}_{3} \mathrm{Fe}(\mathrm{CN})_{6}$ electrolyte. The current density was calculated using the geometrical surface area of the electrode. As shown in Fig. 3(a), the cathodic currents changed little with light illumination. However, the anodic currents increased under illumination where the dark 
current showed good blocking behavior. These results indicate that the basic photovoltaic property of n-type semiconductors remained on the porous InP electrode.

The anodic photocurrents of the various porous electrodes with different $d$ 's of 3.2, 4.8, 7.2, and $9.6 \mu \mathrm{m}$ are compared in Fig. 3(b). In comparison, the photocurrents observed for the planar electrode $(d=0 \mu \mathrm{m})$ were very small and negligible in a range of $0.1 \mathrm{~mA} / \mathrm{cm}^{2}$. On the other hand, the photocurrents of the InP porous electrodes were observed in this current range for all samples, as shown in Fig. 3(b). Furthermore, the anodic currents were found to increase with $d$ whereas the cathodic currents did not change very much.

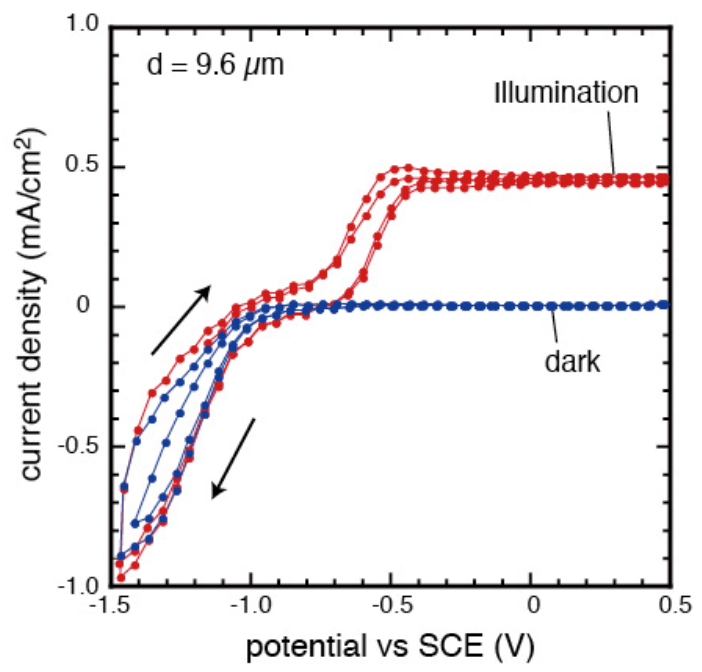

(a)

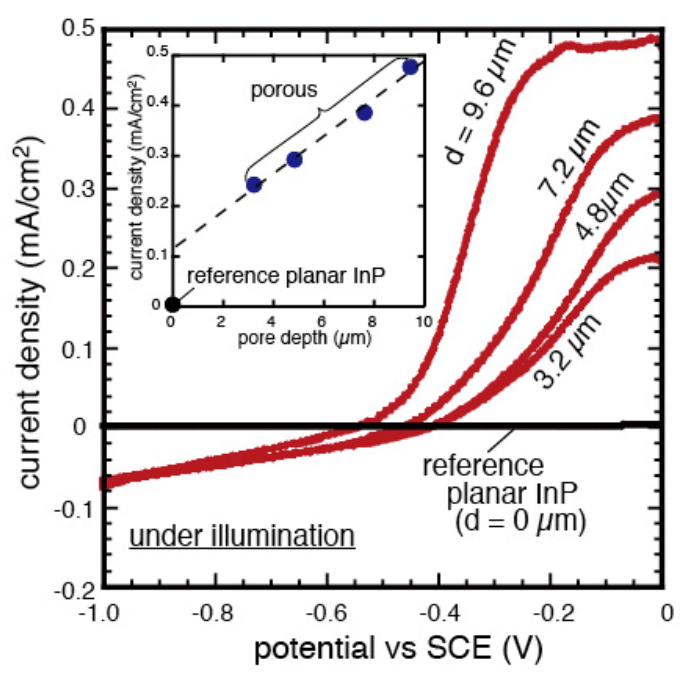

(b)

Figure 3. (a) Cyclic voltammograms measured on InP porous electrode with pore depth, $d$, of $9.6 \mu \mathrm{m}$ in $\mathrm{K}_{3} \mathrm{Fe}(\mathrm{CN})_{6}$ electrolyte. (b) Current-voltage $(I-V)$ characteristics of InP porous structures that have different pore depth, $d$, of 3.2, 4.8, 7.2 9.6 $\mu \mathrm{m}$. Inset shows the correlation between anodic photocurrents measured at applied potential of $0 \mathrm{~V}$ and $d$.

Inset of Fig. 3(b) shows the plot of the anodic photocurrents measured at the applied potential of $0 \mathrm{~V}$ as a function of the pore depth, $d$. This plot showed approximately linear relationships with $d$ as expected because the anode efficiency proportionally increased with the surface area of the inside pore that was enlarged as a linear function of $d$. Similar effects of the enlarged surface area have been reported on the amperometric chemical sensor based on the InP porous structures (15). However, the difference in the photocurrents between the porous electrodes and the reference planar electrode cannot be explained only by the effects of the enlarged surface area. As shown in the inset, the extrapolation value given by the data of the porous electrodes was larger than the experimentally obtained value plotted at $d=0 \mu \mathrm{m}$ for the reference planar electrode.

These results suggest that the porous structures have a larger incident light absorption property than bulk InP. As shown in Fig. 2(b), the extremely low reflectance surfaces were obtained on the porous structures after the PEC etching, where the pores with the enlarged openings were vertically aligned. In such a case, the incident light goes through the porous layer by repeating the absorption on the pore walls due to an effective optical path longer than that of bulk InP. Similar discussion has been also made on the light 
absorption process in nanowire materials (16). Another possible reason for the absorption enhancing is the optical transition involving both surface states (17) and quantum states (18) formed on the pore wall. These additional transitions give extra absorption in a wider energy range than the band gap of bulk InP. Thus, it can be concluded that the efficiency of a photon energy conversion is enhanced in the porous layer, resulting in the large anodic photocurrents increasing with the number of positive holes.

\section{Conclusions}

In conclusion, we have demonstrated that the surface reflectance of InP was significantly reduced by the formation of the porous structures. PEC etching after the pore formation very effectively reduced the reflectance to less than $0.1 \%$ in the visible light range and less than $0.4 \%$ in the UV and near-infrared ranges. Photoelectrochemical measurements on the porous structures revealed that the large anodic photocurrents are obtained on the samples that have low reflectance surfaces with deeper pores. These results can be explained by the two unique features of the InP porous structures: the large surface area inside pores and the large photon absorption in the porous layer.

\section{Acknowledgments}

The work reported here was supported in part by a Grant-in-Aid for young scientists (A) 21686028 and by Grant-in-Aid for Challenging Exploratory Research - 21656078, from the Japanese Ministry of Education, Culture, Sports, Science, and Technology.

\section{References}

1. C. X. Shi, D. Grutzmacher, M. Stollenwerk, Q. K. Wang, and K. Heime: IEEE Trans. Electron Devices, 39, 1028 (1992).

2. F. Zappa, A. Lacaita, S. Cova and, P. Webb: Opt. Lett., 19, 846 (1996).

3. A. Khan, A. Freundlich, J. Gou, A. Gapud, M. Imazumi, and M. Yamaguchi: Appl. Phys. Lett., 90, 233111 (2007).

4. M. F. Schubert, F. W. Mont, S. Chhajed, D. J. Poxson, J. K. Kim, and E. F. Schubert: Opt. Exp., 16, 5291 (2008).

5. P. Jenkins, G. A. Landis, N. S. Fatemi, D. Scheiman, X. Li, and S. G. Bailey: Sol. Energy Mater. Sol. Cells, 33, 125 (1994).

6. N. G. Ferreira, D. Soltz, F. Decker, and L. Cescato: J. Electrochem. Soc., 142, 1348 (1995).

7. N. L. Dmitruk, O. Y. Borkovskaya, I. B. Mamontova, O. I. Mayeva, and O. B. Yastrubchak: Thin Solid Films, 364, 280 (2000).

8. M. G. Boudreau, S. G. Wallace, G. Balcaitis, S. Murugkar, H. K. Haugen, and P. Mascher: Appl. Optics, 39, 1053 (2000).

9. M. R. Hantehzadeh, M. Ghoranneviss, A. H. Sari, F. Sahlani, A. Shokuhi, and M. Shariati: Thin Solid Films, 515, 547 (2006).

10. K. Ramamoorthy, K. Kumar, R. Chandramohan, K. Sankaranarayanan, R. Saravanan, I. V. Kityk, and P. Ramasamy: Opt. Commun., 262, 91 (2006).

11. T. Sato, T. Fujino, and H. Hasegawa: Appl. Surf. Sci., 252, 5457 (2006). 
12. T. Sato, and A. Mizohata: Electrochem. Solid State Lett., 11, H111 (2008).

13. A. Theuwis, and I. E. Vermeir: J. Electrochem. Soc., 146, 1172 (1999).

14. P. Lautenschlager, M. Garriga, and M. Cardona: Phys. Rev. B, 36, 4813 (1987).

15. T. Sato, A. Mizohata, N. Yoshizawa, and T. Hashizume: Appl. Phys. Exp., 1, 051202 (2008).

16. O. L. Muskens, J. G. Rivas, R. E. Algra, E. P. A. M. Bakkers, and A. Lagendijk: Nano Lett., 8, 2638 (2008).

17. H. Fujikura, A. Liu, A. Hamamatsu, T. Sato, and H. Hasegawa: Jpn. J. Appl. Phys., 39, 4616 (2000).

18. T. Sato, T. Fujino, and T. Hashizume: Electrochem. Solid State Lett., 10, H153 (2007). 